\title{
Von den alltäglichsten Sachen zu Abfall für alle Diaristische Praktiken und Unnützes Wissen um 1800, 1900, 2000
}

\section{Kurze Vorüberlegung: Tagebuch und unsicheres Wissen vom Menschen}

Das Tagebuch ist eine Gebrauchsform. Und wie kaum eine Textsorte ist es durchlässig für alle Wissensfelder und zeigt dabei Subjektivität in actu. Durch seine Affinität zu den Wissenschaften vom Menschen ist das Tagebuch ein prädestinierter Ort für unsicheres Wissen; entsprechende Ansätze finden sich in der Forschung zur literarischen Anthropologie sowie zur Poetologie des Wissens. ${ }^{1}$ Und durch seine Tagestaktung, seine Dynamik von Unterbrechung und Neueinsatz kann das Tagebuch der temporalen Verfassung des unsicheren Wissens in besonderer Weise gerecht werden. ${ }^{2}$ In dieser Perspektive lassen sich in der Geschichte des Tagebuchs wichtige literar- und wissenshistorische Zäsuren um 1800, um 1900 und um 2000 ausmachen, und im Folgenden werden solche Diaristen in den Blick genommen, die neue Darstellungsformate für das Wissen vom Menschen erproben: Der Theologe und Anthropologe Johann Caspar Lavater publiziert 1771 das Geheime Tagebuch. Von einem Beobachter Seiner Selbst sowie 1773 des Tagebuches Zweyter Teil und begründet damit sowohl die literarische Gattung als auch eine empirische Quellensorte für die sich ein Jahrzehnt später

1 Vgl. Helmut Pfotenhauer, Literarische Anthropologie. Selbstbiographien und ihre Geschichte - am Leitfaden des Leibes, Stuttgart 1987, S. 81-92; Manfred Schneider, Politik der Lebensgeschichte um 1800 und das autobiographische Wissen im Theoriedesign des 20. Jahrhunderts, in: Joseph Vogl (Hg.), Poetologien des Wissens um 1800, München 1999, S. 267-288.

2 Siehe dazu die in Abgrenzung von der Differenz Wissen/Nichtwissen "graduell und prozesshaft zu denkenden Unterscheidung von Unsicherem und Sicherem Wissen« im Konzept des SNF-Forschungsprojekts Das unsichere Wissen der Literatur: Maximilian Bergengruen/ Peter Schnyder/Hans Georg von Arburg, Das unsichere Wissen der Literatur. Natur, Recht, Ästhetik, http://www.unsichereswissen.ch/fileadmin/pdfs/unsichereswissen-Ausbildungsmodul.pdf [Datum des letzten Zugriffs: 10.08.2014]; Zur temporären Struktur des Tagebuchs siehe: Günter Oesterle, Die Intervalle des Tagebuchs - das Tagebuch als Intervall, in: Helmut Gold/Christiane Holm/Eva Bös/Tine Nowak (Hg.), @bsolut privat? Vom Tagebuch zum Weblog, Heidelberg 2008 (Kataloge der Museumsstiftung Post und Telekommunikation; 26), S. 100-103. 
etablierende Erfahrungsseelenkunde. ${ }^{3}$ Der Erfinder des inneren Monologs und praktizierende Arzt Arthur Schnitzler bereitet die postume Publikation seines umfangreichen, von 1875 bis 1931 entstandenen, diaristischen Lebenswerkes vor, worin er sich im Selbstversuch mit Sigmund Freuds 1900 erschienener Traumdeutung auseinandersetzt. ${ }^{4}$ Der promovierte Psychiater und Pop-Literat Rainald Goetz veröffentlicht sein 1998 bis 1999 online geführtes und anschließend in Buchform überführtes Tagebuch Abfall für alle, in dem er sich mit der elektronisch disponierten Aufnahme und Verarbeitung von Datenpaketen aus allen medial präsenten Wissensfeldern auseinandersetzt, und erhält dafür nicht allein medienwissenschaftliche Aufmerksamkeit, sondern im Jahr 2000 auch den Raabe-Preis für eine innovative Erzählform. ${ }^{5}$ Eine entscheidende Gemeinsamkeit der drei gewählten Autoren besteht darin, dass sie ihre Tagebücher nicht als literarische Werke, wohl aber mit großem Interesse für ihre Poetizität konsequent aus einer diaristischen Praxis heraus konzipieren.

\section{Das Tagebuch als Gebrauchsform}

Bei Nutz und Unnutz handelt es sich, so das Konzept des vorliegenden Bandes, um referenzielle Kategorien des Wissens, die über ihre diskursive Brauchbarkeit definiert sind. ${ }^{6}$ Die Frage danach, ob das Tagebuch als Gebrauchsform eine gattungsspezifische Beziehung zum Unnützen Wissen unterhält, rückt dessen Verfahrensweisen in den Vordergrund. Fokussiert

3 Vgl. Johannes F. Lehmann, Anthropologie, in: Roland Borgards/Harald Neumeyer/Nicolas Pethes/Yvonne Wübben (Hg.), Literatur und Wissen. Ein interdisziplinäres Handbuch, Stuttgart 2013, S. 57-63, hier S. 58-60. Sibylle Schönborn, Das Buch der Seele. Tagebuchliteratur zwischen Aufklärung und Kunstperiode, Tübingen 1999 (Studien und Texte zur Sozialgeschichte der Literatur; 68), S. 86-126.

4 Vgl. Marcus Krause, Psychologie, in: Roland Borgards/Harald Neumeyer/Nicolas Pethes/ Yvonne Wübben (Hg.), Literatur und Wissen. Ein interdisziplinäres Handbuch. Stuttgart 2013, S. 131-141, hier S. 137-140; Leo A. Lensing, Nachwort, zu: Arthur Schnitzler, Träume. Das Traumtagebuch 1875-1931, Göttingen 2012. S. 407-458.

5 Vgl. Rainald Goetz/Diedrich Diederichsen, mehr, mit einer Einleitung von Joseph Vogl, am 3.5.2012, in: Fortsetzung folgt... Formate des Seriellen in den Künsten und Medien. [Mosse]Lectures an der Humboldt-Universität zu Berlin im Sommersemester 2012, http://www.mosse-lectures.de/web/index.php/de/content/archive/SS12.html [Datum des letzten Zugriffs: 10.08.2014]. Elke Siegel, Remains of the Day. Rainald Goetz's Internet Diary Abfall für alle, in: The Germanic Review: Literature, Gulture, Theory, 81/3 (2006), S. 235-254.

6 Vgl. Jill Bühler/Antonia Eder, Einleitung zum vorliegenden Band. 
die literaturgeschichtliche wie die wissenspoetologische Forschung zumeist die Ich-Form und somit das Feld des unsicheren Wissens, wie einleitend skizziert, so setzt die folgende Analyse bei der Tages-Form an, um das Unnütze Wissen in den Blick zu bekommen.

Ein Tagebuch ist durch einen unhintergehbaren Rhythmus definiert. Darin unterscheidet es sich grundsätzlich von der Autobiographie, die retrospektiv ein Ich entwirft, ob nun eingebettet in große Erzählbögen oder episodisch arrangiert. Das diaristische Schreiben hingegen ist im Tagestakt organisiert, was sich formal in der "Texteinheit TAG « abbildet. ${ }^{7}$ Und anders als in der Chronik, die nach inhaltlichen Kriterien selektiert und nur eine bestimmte Art von Ereignissen kalendarisch notiert, ist im Tagebuch jeder Tag einen Eintrag wert. Die unterschiedliche Länge der Einträge wie auch Auslassungen unterliegen gleichsam diesem Tagestakt und machen den besonderen rhythmischen Reiz dieser Schreibform aus.

Diese Öffnung des Textes zum Alltäglichen macht ihn durchlässig für solcherart Wissen, das entweder vom Diaristen selbst - im Schreibakt oder in der Relektüre - oder auch von den externen Lesern als epistemisch unnütz und ästhetisch uninteressant eingestuft wird. Im Verlauf des 19. Jahrhunderts wird zur Beschreibung des Phänomens der pejorativ gefärbte Begriff banal aus dem Französischen übernommen, der sich seinerseits von dem altfranzösischen Rechtswort ban ableitet, das gemeinnützige Güter bezeichnet. Insbesondere dem veröffentlichten und somit seinem subjektiven Gebrauchskontext enthobenen Tagebuch wird seine hohe Affinität zum Banalen vorgeworfen. Banalität bezeichnet nicht nur das Gemeine auf der Ebene des Gegenstandes, sondern auch das Repetitive und Phrasenhafte auf der Darstellungsebene. ${ }^{8}$ Muss sich das Tagebuch immer auch dazu verhalten, dass es für andere Wissensfelder nicht brauchbare Informationen aufnimmt, so konstituiert sich darüber hinaus im Prozess des Schreibens selbst ein Feld Unnützen Wissens. Es handelt sich um Wissen, das innerhalb der jeweiligen diaristischen Praxis keinen Gebrauchswert erhält, und das nur in Relation zum expliziten oder impliziten Programm des jeweiligen Tagebuchs als unnütz taxierbar ist.

7 Arno Dusini, Tagebuch. Möglichkeiten einer Gattung, München 2005, S. 83-108. Dieser Ansatz hat sich zudem in der vergleichenden Analyse von Tagebüchern und Weblogs bewährt: Helmut Gold/Christiane Holm/Eva Bös/Tine Nowak (Hg.), @bsolut privat? Vom Tagebuch zum Weblog, Heidelberg 2008.

8 Vgl. Julia Genz, Diskurse der Wertung. Banalität, Trivialität und Kitsch, München 2011, S. 30-33. 
Im Folgenden seien drei zentrale Gebrauchsfunktionen sowie die ihnen entsprechenden Verfahren des Diaristischen skizziert, welche sich innerhalb eines Tagebuchs freilich selten in Reinform, sondern in unterschiedlichen Konstellationen und Konjunkturen finden: ${ }^{9}$

Für die meisten Tagebücher ist die Erinnerungsfunktion zentral. Der archivierende Zugriff transformiert das Tagesgeschehen in verfügbare Vergangenheit. Im Unterschied zu anderen autobiographischen Verfahren bietet der Kalender das Raster für die Einträge, die Datumsziffern sind Teil des Textes. Besonders augenscheinlich ist dieser Zusammenhang in den sogenannten Schreibkalendern, dem dominierenden Tagebuchformat vom 17. bis ins 19. Jahrhundert, das jedem Tag ein Textfeld einräumt. ${ }^{10}$

Die Funktion der Selbstformung hingegen ist weniger auf die Retrospektion als vielmehr auf die Zukunftsgestaltung gerichtet. Im Verfahren des Bilanzierens werden die Tageserträge gemessen, mitunter in Monats- und Jahreserträgen gebündelt und mit Blick auf die Selbstoptimierung ausgewertet. Die zentrale Referenzform findet sich im Rechenbuch, das seit dem 16. Jahrhundert auch als »Tagbuch" oder »Diarium« bezeichnet wurde. ${ }^{11}$

Die Funktion der Verortung prägt die Notation der offenen Verläufe. Als strukturierendes Prinzip findet sie sich vor allem in Tagebüchern von Reisen, ebenso im Sinne von Lebensreisen, oder auch von Arbeitsprozessen, insbesondere in Textwerkstätten. Hier werden Abläufe notiert, Varianten ausprobiert, Positionen eingetragen und Etappen kommentiert. Die Referenzform ist das Logbuch, das in den Neologismus des Weblogs bzw. Blogs eingegangen ist, was auf die Navigationsverfahren in der elektronischen Informationsflut anspielt.

Quer zu diesen drei Verfahren - dem kalendarischen Archivieren, dem Bilanzieren und dem Navigieren - sind die im Tagebuch, wie aber auch in anderen autobiographischen Schreibformen aufzufindenden, Selbsttechniken zu ergänzen. ${ }^{12} \mathrm{Zu}$ nennen sind vor allem Selbstbeobachtung und -reflexion, die sich nahezu automatisch in der Dissoziierung von schreibendem, ge-

9 Die folgende Skizze der Gebrauchsfunktionen basiert auf der ausführlichen Darstellung im oben genannten Ausstellungskatalog: Christiane Holm, Montag Ich. Dienstag Ich. Mittwoch Ich. Versuch einer Phänomenologie des Diaristischen, in: Helmut Gold/Christiane Holm/Eva Bös/Tine Nowak (Hg.), @bsolut privat? Vom Tagebuch zum Weblog, Heidelberg 2008, S. 10-50.

10 Vgl. Harald Tersch, Schreibkalender und Schreibkultur. Zur Rezeptionsgeschichte eines frühen Massenmediums, Graz-Feldkirch 2008, S. 75-80.

11 Christiane Holm, Montag Ich. Dienstag Ich. Mittwoch Ich, S. 15.

12 Vgl. Michel Foucault, Technologien des Selbst, hg. von Luther H. Martin, Frankfurt a.M. 1993, S. 24-62. 
schriebenem und adressiertem Ich einstellen, sowie die Selbsterleichterung der Katharsis, die das Tagebuch bei konkretem Anlass in psychohygienischer Absicht nutzt. ${ }^{13}$

Im Folgenden wird nun an den eingangs genannten, epochalen Fallbeispielen der Tagebücher von Lavater, Schnitzler und Goetz untersucht, wie Unnützes Wissen erstens aus der Logik der jeweiligen Gebrauchsfunktion heraus entsteht, wie es zweitens innerhalb der jeweiligen Textpraxis beobachtet und reflektiert wird und welche Rolle dieses drittens im Programm des jeweiligen diaristischen Projektes spielt.

III. 1800: Lavater

Die beiden ersten zu Lebzeiten eines Autors gedruckten Tagebücher, das 1771 erschienene Geheime Tagebuch, das den 1. bis 31. Januar 1769 umfasst, und die 1773 daran anschließenden Unveränderten Fragmente aus dem Tagebuche, die ausgewählte Einträge von November 1772 bis Juni 1773 enthalten, folgen, bei gleich bleibenden Inhalten aus häuslichem Arbeits- und Familienleben, völlig unterschiedlichen Programmen.

Das Geheime Tagebuch wird im Vorbericht des anonymen Herausgebers über seinen Nutzen für die fromme Lebenspraxis definiert:

So viel ist gewiß [...], daß eine getreue und umständliche moralische Lebensbeschreibung des gemeinsten und unromanhaftesten Menschen unendlich wichtiger, und zur Verbesserung des Herzens ungleich tauglicher ist, als der sonderbarste und interessanteste Roman. Es giebt immer tausend Menschen, denen die erstere, gegen Einen, dem der letztere einen wahren, einen dauerhaften moralischen Nutzen gewähren kann. Wenige sind berufen, Helden auf dem öffentlichen Schauplatze der Welt, aber alle Helden in der häuslichen Tugend zu werden. ${ }^{14}$

Das Tagebuch setzt in der Silvesternacht ein mit der Formulierung einer Versuchsanordnung, den »Täglichen Grundsätzen «. ${ }^{15}$ In diesen zwölf Punkten werden Tageszeiten und andere Anlässe für Bibellektüre und Gebete genauestens festgelegt, weitere Zielvorstellungen sind, den Mitmenschen in konkreten Lebenssituationen "nützlich zu seyn" und schließlich

13 Vgl. Peter Boerner, Tagebuch, Stuttgart 1969, S. 20-23.

14 Anon. [Johann Caspar Lavater], Geheimes Tagebuch. Von einem Beobachter Seiner Selbst, [hg. von anon. Georg Joachim Zollikofer], Leipzig 1771, Vorbericht des Herausgebers [Hervorh. im Original], S. 5 f.

15 Ebd., Einleitung. Januar 1769, S. 14-17. 
allabendlich die unerfüllten Punkte sowie die Lernerfolge »unter dem lauten Beyfalle meines Gewissens" aufzuzeichnen. ${ }^{16}$ Dieses Bilanzieren wiederholt sich idealiter täglich, monatlich und jährlich und arbeitet dem letzten Tag der "Rechnung über mein Leben" zu. ${ }^{17}$ Es handelt sich um ein Musterbeispiel der frommen Selbstformung, in dem das von Gott ohnehin geführte Verzeichnis von Nutz und Unnutz im Buch des Lebens durch das Diarium in die eigene Lebenspraxis vorverlegt wird. ${ }^{18}$ Dieser Tagebuch-Typus gründet auf dem theologischen Diktum von der "Auskauffung der Zeit« und findet sich bereits im englischen Puritanismus wie im Halle'schen Pietismus in voller Ausprägung. ${ }^{19}$

Bei einer vergleichsweise negativen Bilanz, bedingt durch eine Reihe von Zerstreuungen, konkret: einen Friseurbesuch, ein Gastmahl und eine Schlittenfahrt, wechselt der Diarist von "aller Beschreibung in die komprimierende "Zeichnung ${ }^{20}{ }^{20}$ Nicht verzichtet wird jedoch auf die Notation von Tages- und Uhrzeit, so dass die Logik der Buchführung erhalten bleibt. Im ersten Bild ist zudem die Uhr platziert, was in barocker Emblematik mit den beiden en face ins Bild gesetzten und somit aufeinander bezogenen Gesichtern von Totenschädel und Frisiertem an die "Auskauffung der Zeit» erinnert.

16 Ebd., S. 15; S. 17.

17 Ebd., S. 15.

18 Schönborn kontextualisiert das "strenge, didaktische Konstruktionsschema" dieses "Romans des Gewissens" in ihrer Studie über Tagebücher des 18. Jahrhunderts mit anderen christlichen, vornehmlich pietistisch inspirierten Diarien. Schönborn, Das Buch der Seele, S. 96.

19 August Herrmann Francke hat den deutschsprachigen Terminus 1713 in seiner Predigt Der rechte Gebrauch der Zeit eingeführt. Christiane Holm, Versuch einer Phänomenologie des Diaristischen, S. 15. Vgl. dazu Shirley Brückner, Kulturen der Berechenbarkeit. Religiosität und Lebensführung im Pietismus, Diss. Halle 2010, S. 79-112, http://digital.bibliothek.uni-halle.de/hs/content/titleinfo/2285797 [Datum des letzten Zugriffs: 15.09.2015].

20 Johann Caspar Lavater, Geheimes Tagebuch, 17ten Januar, o. S. (zwischen S. 174 und 175). 


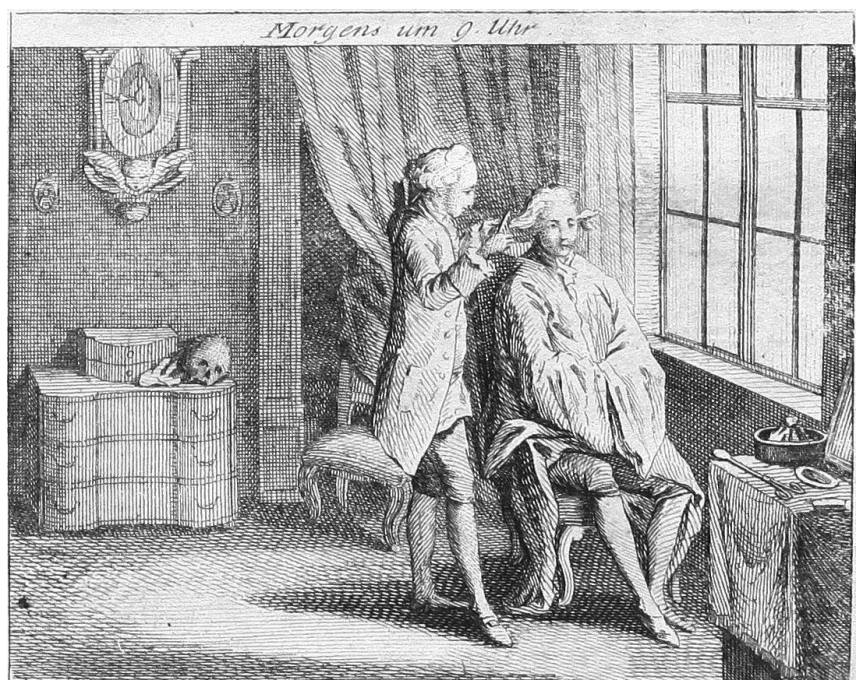

Abb. 1 Morgens um 9 Uhr, Kupferstich, 5,4 x 6,9 $\mathrm{cm}^{21}$

\section{Am Folgetag wird die skizzierte Bilanz schriftlich nachbereitet:}

[...] - der Verlust eines Tages - welch ein unersetzlicher Verlust! - wer giebt mir die Freyheit und das Recht, einen Tag, der meines Gottes ist, wegzuwerfen? [...] Gesetzt nun auch, daß alles, was ich gethan habe, unschuldig gewesen, und alles, was ich unterlassen habe, nach dem Urtheile aller Sittenlehrer mit Unschuld unterlassen worden wäre - wird mir nicht immer noch die Kränkung übrig bleiben, zu denken, dieser Tag hätte dennoch auf eine weit nützlichere, für mich und andere noch in der Ewigkeit gesegnete Weise zugebracht werden können? - Ein Kaufmann, der an einem Tage, da er tausend Thaler hätte gewinnen können, bloß drey oder vier gewonnen hat, wird sich schwerlich davon überreden können, daß er einen guten Tag gehabt, wenn gleich vielleicht ein anderer, der gewohnt ist, in vielen Tagen wenig oder nichts zu gewinnen, diese unbeträchtliche Summe groß genug finden wird. ${ }^{22}$

Die Sünde besteht hier nicht in der tugendlosen Tat, sondern im Wegwerfen der Zeit, in der Ignoranz ihres Kapitalwertes für potenziell nützliche Handlungen. Nicht nur die Lockungen der Welt, sondern gerade auch die häuslichen Tätigkeiten wie das bummelnde Entfachen des Kaminfeuers

21 Illustration aus: [anon. Johann Caspar Lavater], Geheimes Tagebuch. Von einem Beobachter Seiner Selbst, Leipzig 1771, Zweigbibliothek Europäische Aufklärung, Sign. Dd 2767d, (C) Universitäts- und Landesbibliothek Halle.

22 Ebd., Den achtzehnten Januar [1769], S. 178f. 
oder unnütze Plaudereien können als Minusgeschäft verbucht werden, und so wundert es nicht, dass schließlich das Tagebuch selbst unter den Verdacht der "Zerstreuungssucht« gerät. ${ }^{23}$ Denn, so beobachtet der Diarist sein Tun, es verleitet dazu, die »Thorheiten" schreibend zu bekennen anstatt sie handelnd zu verbessern, so dass das Notieren des Unnützen paradoxerweise zusätzlich in die Negativbilanz eingerechnet werden muss - bei dieser Einsicht wird der Eintrag umgehend untergebrochen, um sich erst am Folgetag mit positiven Bilanzen zurückzumelden. ${ }^{24}$

Die entstandenen Aporien des Mediums selbst können jedoch im letzten Eintrag behoben und die unbeirrt fortgeführte Praxis retrospektiv legitimiert werden, indem Nutz und Unnutz in neuer Weise am "großen Endzweck « eines frommen Lebens ausgerichtet werden. ${ }^{25}$ So resümiert der Diarist, dass die "ernsthaftesten Sachen« vielleicht "Zerstreuung für mich werden« können, während »die alltäglichsten und gleichgültigsten Sachen« sich durchaus "auf diesen Endzweck lenken« lassen. ${ }^{26}$ So gibt es letztlich keine nützlichen und unnützen Sachen, wie es die vorangestellten »Grundsätze« listen, sondern allein eine nutz- oder unnutzbringende Praxis.

In seiner zweiten Tagebuchpublikation, den Unveränderten Fragmenten aus dem Tagebuche eines Beobachters seiner Selbst, gibt sich Lavater, inzwischen hatte er sich einen Namen als Autor gemacht, einleitend in einem Brief an den anonym bleibenden Herausgeber zu erkennen. ${ }^{27}$ Das Buch bezieht seine Spannung daraus, dass sich Herausgeber und Diarist in Folge nicht mehr demselben Ideal verpflichten. Während der Herausgeber in der Vorbemerkung und den mitgeführten Anmerkungen an dem Nützlichkeitspostulat festhält, verfolgt der Diarist ein verändertes Programm, indem er den Spielraum für das Vergnügen erweitert. So dient der Gedanke, der »von einigen verständigen Lesern abgeborget [wird], daß ich noch nichts nützlicheres geschrieben habe, und nichts nützlicheres schreiben werde, als ein solches Tagebuch", der Legitimation der letztlich nicht überprüfbaren Variante, dass das Buch »im höchsten Grade erdichtet wäre« und wie ein "moralischer

23 Ebd., Den achten Januar [1769], S. 98f.

24 Vgl. ebd., S. 99.

25 Ebd., S. 259.

26 Ebd.

$27 \mathrm{Zu}$ den darstellerischen Konsequenzen der enthüllten Anonymität siehe: Stephan Pabst, Schamlose Beobachtung. Lavaters Geheimes Tagebuch, in: Ders. (Hg.), Anonymität und Autorschaft. Zur Literatur- und Rechtsgeschichte der Namenlosigkeit, Berlin/New York 2011, S. 177-204. 
Roman« wirkt. ${ }^{28}$ Und konsequenterweise wird im Folgenden der Wert des veröffentlichten Tagebuchs an der - von Lavater für die schöne Literatur selbst schon verabschiedeten - wirkungsästhetischen Formel von prodesse und delectare ausgerichtet. ${ }^{29}$

Die Unveränderten Fragmente erscheinen ohne vorgeschaltetes Programm, erst am 1. Januar 1773 wird die Versuchsanordnung nachgereicht:

Ein vollständiges Tagebuch zu machen, dazu habe ich keine Zeit mehr; ich will also nur, so viel meine übrigen Augenblicke, die ich nicht besser anwenden kann, zulassen, meine merkwürdigsten Stunden, Beschäftigungen, Situationen, Vorfallenheiten, Empfindungen, Schwachheiten, Uebereilungen, Fehler, so kurz, und für mich lehrreich, als es mir möglich seyn wird, aufzuzeichnen; desto umständlicher, je mehr ich Zeit habe; desto kürzer, je weniger. Es soll mich nicht unruhig machen, wenn ich daran gehindert werde; ich will mich auch in diesem Stücke ohne alle Ängstlichkeit nach der Schickung der Fürsehung kindlich und einfältig bequemen. ${ }^{30}$

Diese Neujustierung im Zeichen der Fürsehung, der Verzicht auf die Selbstorganisation zugunsten der kindlichen Einlassung auf das göttlich gelenkte Tagesgeschehen, wird tatsächlich zum Zentralthema des Buches und entsprechend kritisch von dem Herausgeber in den Anmerkungen kommentiert. Es führt schließlich so weit, dass solche im vorangegangenen Tagebuch als Zerstreuung gekennzeichneten Tätigkeiten nun eine eigene Aufmerksamkeit erhalten:

Mein Oncle kam. Eine Stadtgeschichte. Ich hörte sie mit der Aufmerksamkeit an, als wenn ich sonst nichts in der Welt zu hören oder zu thun hätte, und es wurden dadurch manche gute, mit der Zeit brauchbare Reflexionen in mir veranlaßt. Ueberhaupt bemerke und erfahre ich mit einer Zuverlässigkeit, die mir keinen Zweifel übrig läßt, daß dieses einfältige Hören bei den allergleichgültigsten Reden, diese Unterwerfung unter alle Fügungen der göttlichen Fürsehung auch bei den alltäglichsten Dingen dieser Welt eine unvergleichliche Sache, eine vortreffliche Schule der menschenliebenden Demuth, ein herrliches Mittel ist, andern zu nützen und von andern Nutzen zu haben. - - - ${ }^{31}$

Es zeichnet sich hier ein neuer Blick auf das Alltägliche ab, wobei sich im Tagebuch »jene Umstellung von Dogmatismus auf Empirie« abbildet, die

28 Johann Caspar Lavater], Unveränderte Fragmente aus dem Tagebuche eines Beobachters seiner Selbst; oder des Tagebuches Zweyter Theil, nebst einem Schreiben an den Herausgeber desselben, [anon. GeorgJoachim Zollikofer (Hg.)], Leipzig 1773, S. XXVIII.

29 Vgl. ebd., S. XIV, XXIX.

30 Ebd., Freytag der 1. Jenner 1773, S. 69.

31 Ebd., Sonntag der 10. Jenner 1773, S. 87. 
die "Politik des anthropologischen Wissens" strukturiert. ${ }^{32}$ Rangierte das Unnütze im ersten Tagebuch eindeutig unter der teuflischen Zerstreuung, so wird es hier der göttlichen Fürsehung zugerechnet. Dabei wird die zeitliche Ausrichtung des bilanzierenden Schreibens auf das Lebensende abgelöst durch den mitunter emphatischen Gegenwartsbezug des situativnavigierenden Schreibens. Die Leser der beiden Tagebücher können nachvollziehen, wie Nutz und Unnutz im rhythmisierten Schreibprozess neu definiert und dabei von absoluten zu relationalen Größen frommer Lebensführung werden.

\section{1900: Schnitzler}

Schnitzler begann sein Tagebuch als 17-Jähriger und führte es regelmäßig bis zum Lebensende. Dabei experimentierte er im ersten Jahrzehnt mit verschiedenen Verfahren: Das Bilanzieren verwendet er etwa für seine Opernund Theaterbesuche, das Navigieren für das Liebesleben mit verschiedenen Akteurinnen. Einen Großteil seiner frühen Bücher hat Schnitzler später vernichtet, denn "seinen Zweck hat das Geschreibsel genugsam erfüllt, indem es mich zur Zeit seines Entstehens beruhigte «. ${ }^{33}$ Sein Tagebuch fungierte folglich als psychohygienisches Instrument, als »[h]older Spucknapf meiner Stimmungen und Verstimmungen ${ }^{34}$ Zudem problematisiert der Diarist, dass weniger die Brisanz als vielmehr die Banalität der rausgespuckten Einträge Grund für ihre Vernichtung ist, wie er am 1. Februar 1882 bemerkt:

Nichts neues.- Man geht eben ab und zu ins Theater - oder besucht eine Vorlesung - oder liest eine Novelle - oder mikroskopiert,- oder studiert - oder geht ins Café plaudert über Dinge, die man will und nicht hat, wobei man sich ärgert, oder über Dinge, die man nicht will und doch hat, was einen auch ärgert, oder über Dinge, die man will und hat - die einem folglich eine Stunde später gleichgiltig sind ... Um Himmelswillen! Flammen! Glanz! Entzücken.

... Ob es nicht beinahe gescheidt wäre, sich zu verlieben ... Und doch, wie kindisch, wie kleinlich: Ich denke, hab ich einmal mein lästiges Rigorosum hinter mir, so im Mai, Juni, wird sich wieder mal (wieder?) ungehindert leben lassen. Ah, blöd,

32 Manfred Schneider, Politik der Lebensgeschichte um 1800, S. 271f.

33 Arthur Schnitzler, Tagebuch 1879-1892 [Bd. 1], hg. von Werner Welzig unter Mitwirkung von Peter Michael Braunwarth, Susanne Pertlik und Reinhard Urbach von der Kommission für literarische Gebrauchsformen der Österreichischen Akademie der Wissenschaften, Wien 1987, 16/10 Sonntag Abend [1881], S. 112.

34 Ebd., 27/6 Sonntag früh [1880], S. 66. 
lächerlich - wozu ich eigentlich das dumme Zeug alles hinkritzle, Blatt für Blatt langweilt mich schon jetzt und wird später sicher verbrannt. ${ }^{35}$

In solchen Selbstbeschreibungen des Tagebuchs werden Textpraxis und Text prinzipiell voneinander geschieden: Movens ist der psychohygienische Nutzen des "Spucknapfes" im Schreibakt, sein Inhalt jedoch ist nicht lesenswert, der aufgezeichnete Text wird folglich nicht mehr gebraucht, er ist nutzlos und kann entsorgt werden.

Um 1900 findet Schnitzlers Tagebuch zu einer gänzlich anderen Form, die sich fortan stabilisiert: Im nüchternen Stenogrammstil, durchsetzt mit zahlreichen Abkürzungen, werden die wichtigsten Erlebnisse des Tages notiert. Teil dieses archivierenden Verfahrens ist die exzessive Relektüre, die nicht nur zahlreiche Spuren wie Hervorhebungen und Kommentare in den Manuskripten hinterlässt, sondern sich auch in verschiedenen thematisch-chronikal ausgerichteten Exzerpten manifestiert. Testamentarisch verfügte Schnitzler die Publikation seines etwa 8000 Manuskriptseiten umfassendes Lebenswerkes nach seinem Tode, wobei es »in keiner Weise verfälscht, [...] also nicht gemildert, gekürzt, oder sonstwie verändert« werden dürfe. ${ }^{36}$ In den 1920er Jahren unterzog Schnitzler wieder einmal sein gesamtes diaristisches Werk einer thematisch perspektivierenden Relektüre und diktierte seiner Sekretärin Frieda Pollak alle bis dahin verzeichneten über 500 Träume, die in der Regel von Ausdeutungen begleitet sind, in denen sich Hinweise auf eine fachlich-informierte Auseinandersetzung mit Freuds Psychoanalyse finden. ${ }^{37}$ Bezeichnenderweise wird Schnitzlers frühe Lektüre der Traumdeutung im März 1900 nicht im Kontext des Tages-, sondern des Traumgeschehens verzeichnet: "Traum, dass ich in Uniform mit Zivilhosen (wie im Traumdeutungsbuch von Freud gelesen), aber doch unentdeckt von Kaiser Wilhelm II, dem ich begegne, von einem Tor unter den

35 Ebd., 1/2 Mittwoch Abend [1882], S. 121.

36 Arthur Schnitzler, Nachlassverfügung vom 16.8.1918, zit. nach Werner Welzig, Zur Herausgabe von Schnitzlers Tagebuch, in: Arthur Schnitzler, Tagebuch 1879-1892, hg. von Werner Welzig unter Mitwirkung von Peter Michael Braunwarth, Susanne Pertlik und Reinhard Urbach von der Kommission für literarische Gebrauchsformen der Österreichischen Akademie der Wissenschaften, Tagebuch 1909-1912, Wien 1981, S. 7-33, hier S. 15.

37 Leo A. Lensing, Nachwort, in: Arthur Schnitzler, Träume. Das Traumtagebuch 18751931, hg. von Peter Michael Braunwarth/Leo A. Lensing, Göttingen 2012, S. 407-458, hier S. 417-434. Zu Schnitzlers psychologisch informiertem literarischen Beitrag zur Kulturgeschichte des Traums: Peter-André Alt, Der Schlaf der Vernunft. Literatur und Traum in der Kulturgeschichte der Neuzeit, München 2002, S. 345-348. 
Linden ins andere gehe. ${ }^{38}$ Der Diarist führt die Auseinandersetzung mit Freuds Traumdeutung als intensives Lektüreerlebnis eines Textes mit prägnanten Motiven und ignoriert das naheliegende Verfahren der Ausdeutung. Schnitzler gilt, wie nicht zuletzt die zahlreichen Tagebucheinträge belegen, als einer der frühesten Freud-Leser und erkennt auch dessen Rolle als Diskursbegründer an. Seine Auseinandersetzung bleibt jedoch kritisch, insbesondere gegenüber selbstreferenziellen Begründungsverfahren, die er in der Traumdeutung findet. Gerade mit Blick auf die methodisch zentrale Frage nach den Tagesresten verbleibt Schnitzler dezidiert beim vorfreudianischen Forschungsstand. Unumstritten ist, so fasste Friedrich Wilhelm Hildebrandt 1875 zusammen, »das Merkwürdige, daß der Traum seine Elemente in der Regel nicht aus den großen und tiefgreifenden Ereignissen, nicht aus den mächtigen und treibenden Interessen des vergangenen Tages, sondern aus den nebensächlichen Zugaben, sozusagen aus den wertlosen Brocken [...] nimmt ${ }^{39}{ }^{39}$ Freud widerspricht der damit verbundenen Folgerung, dass der Traum "psychische Tätigkeit an läppisches Material verschwendet« und bewertet derartige Banalitäten völlig neu, indem er sie als »Stellvertretung für das psychisch [W]ertvolle« ausweist und »die Tatsache, daß der Trauminhalt Reste von nebensächlichen Erlebnissen aufnimmt, als eine Äußerung der Traumentstellung [deutet] «. ${ }^{40}$ Schnitzler zweifelt generell an dem Status des Unbewussten und der verborgenen Macht der unbewussten Wünsche, sehr wohl aber sieht er strukturelle Analogien von Traum- und Textarbeit. In seinen diaristischen Traumaufzeichnungen plausibilisiert er immer wieder aufs Neue, wie die Inhalte mit den randständigen Erlebnissen des Tages, zu denen insbesondere Lektüren und Schreibarbeiten zählen, verbunden sind. Im Subtext seiner Auslegungen läuft die Kritik an Freuds Traumdeutung häufig mit, wie abschließend an einem Eintrag vom 17. März 1927 gezeigt sei:

Traum. Alserstrasse (wie so oft Krankenhausgegend), eilig, an einem Laden vorbei, - ja richtig - muss mir hier Bleistifte kaufen. (Deutung: Der überflüssige Kauf neulich durch Frau v. Klimbacher [Haushälterin]).

38 Arthur Schnitzler, Träume. Das Traumtagebuch 1875-1931, hg. von Peter Michael Braunwarth/Leo A. Lensing, Göttingen 2012, 26.3. [1900], S. 25f.

39 Friedrich Wilhelm Hildebrandt, Der Traum und seine Verwertung für's Leben, 1875, zit. nach Sigmund Freud, Die Traumdeutung. Studienausgabe, Bd. II, hg. von Alexander Mitscherlich/Angela Richards/James Strachey, Frankfurt a.M. 2000, S. 45.

40 Sigmund Freud, Die Traumdeutung, S. 187; S. $189 f$. 
Gehe hinein. Ältrer Verkäufer, bekannter Herr - (jetzt eben weiss ich, dass es der Trafikant vom Kiosk war, der im Vorjahr starb); ich denke: der ist doch jünger als ich und ist alt. Ich komme mir nicht alt vor! [Schnitzler wird bald 65] - Ich verlange meine gewöhnlichen Kohinoor B. [Markenbleistifte] - Nicht vorrätig. Ich frage nach den Unterschieden. Er weist einen auffallend dicken Bleistift vor; der Verkäufer sagt: durch das Spitzen gehe etwa $25 \%$ verloren; ich sage: 'Ließe sich das nicht verwerten, so wie der Dichter Abfälle von Erlebnissen verwertet aus seelischer Ökonomie? ${ }^{41}$

Die als Deutung ausgewiesene Passage dringt nicht in die Tiefe des Subjekts vor und ignoriert zudem die Freud'sche Steilvorlage zur Traumsymbolik des Bleistifts, mit der Schnitzler sich drei Jahre zuvor an anderer Stelle auseinandersetzte. ${ }^{42}$ Vielmehr notiert er lakonisch den Befund eines Tagesrestes von einem überflüssigen Bleistiftkauf und formuliert dabei eine Deutungstheorie in engem intertextuellen Bezug zur Traumdeutung, die gerade nicht auf das "psychisch [W]ertvolle" zielt, sondern an die Figur von Rest und Abfall rückgekoppelt bleibt: Steht auch das "poetogene Potential des Traumes" außer Frage, ${ }^{43}$ so wird es gerade nicht in seinem psychischen Nutzen im Sinne der Freud'schen "seelischen Ökonomie« verstanden, sondern als produktionsästhetisches Phänomen betrachtet, indem das überschüssige Material in seinen semantischen Eigendynamiken ernst genommen wird. So gesehen besteht der eigentliche Nutzen des Traumtagebuchs darin, die Banalitäten, die selbst der archivierende Tagebucheintrag ausblendet, wieder sichtbar zu machen und dabei ihre Reichweite bis ins poetische Zentrum des träumenden und dichtenden Subjekts zu verfolgen.

41 Arthur Schnitzler, Träume, 17.3. [1927], S. 232.

42 Freud hatte den Bleistift als Phallussymbol in seinen Vorlesungen zur Einführung in die Psychoanalyse beschrieben, die er Schnitzler 1922 schenkte. 1924 führte Schnitzler in seinen Aufzeichnungen Über Psychoanalyse aus, dass derartige Objekte »willkürlich« für Symbole eingesetzt sind und sich in der Traumdeutung am konkreten Material im "circulosis vitiosus« selbst bestätigen. Sigmund Freud, Vorlesungen zur Einführung in die Psychoanalyse (1916-1917), Studienausgabe, Bd. I, hg. von Alexander Mitscherlich/Angela Richards/ James Strachey, Frankfurt a.M. 2000, S. 101-241, hier S. 164. Arthur Schnitzler, Über Psychoanalyse (1924), in: Thomas Anz/Oliver Pfohlman (Hg.), Psychoanalyse in der literarischen Moderne, Eine Dokumentation, Bd. 1, Marburg 2006, S. 176-178, hier S. 177.

43 Manfred Engel, Jeder Träumer ein Shakespeare? Zum poetogenen Potential des Traumes, in: Ders./Rüdiger Zymner (Hg.), Anthropologie der Literatur. Poetogene Strukturen und ästhetisch-soziale Handlungsfelder, Paderborn 2004 (Poetogenesis. Studien und Texte zur empirischen Anthropologie der Literatur; 2), S. 102-117. 


\section{2000: Goetz}

Mit "Los gehts" beginnt der Text in medias res an einem Mittwoch. Im Verlauf der Woche kommt die Idee auf "geil wäre natürlich auch, zu sagen: Sonntag: Ruhetag [...] Abfall gibts nur an Bürotagen - das wäre doch lustig - entstehen dann so Wochenpäckchen «. ${ }^{44}$ Und tatsächlich setzt der Diarist am kommenden Sonntag aus und beginnt in der darauf folgenden Woche mit einem von nun an gültigen Zählsystem. Nach sieben Wochen lässt er dann einen Teil »I« beginnen, und nun zeichnet sich der Plan ab, das Projekt in sieben solcher Teile von je sieben Wochen von je sieben Tagen zu realisieren. Neben dem schlichten, oft kommentierten Datieren bietet dieses parallel geführte, fortlaufende, dreizügige Zählsystem, das jeden Tag innerhalb des Projektes positioniert, einen ständigen und nicht selten emphatisch gefeierten Orientierungspunkt: »Der Tag ist ein göttliches Maß«. ${ }^{45}$

Neben dieser in actu entwickelten Zeitordnung gewinnt auch der experimentelle Status des Textes an Kontur: Das im Februar einsetzende Arbeitstagebuch soll online die für das kommende Sommersemester unter dem Titel Praxis angekündigte Frankfurter Poetikvorlesung vorbereiten und begleiten. In Abfall soll nun im Tagestakt alles das erscheinen, was keinen Eingang in das Vorlesungsskript findet, was also nicht genutzt wird. Sehr genau befolgt der Diarist in den ersten Wochen diese Regel, mit der Konsequenz, dass er mitunter ärgerlich das dort Publizierte als nun für die Vorlesung nicht mehr nutzbar betrachtet. Im Laufe der kommenden Wochen geraten beide Formate in ein "Konkurrenz«-Verhältnis: "Der Aufstand der Baustelle. ${ }^{46}$ Wie erfolgreich der Aufstand verläuft, dokumentiert sich schließlich darin, dass die Vorlesungsskripte für Praxis in Abfall veröffentlicht werden.

Dass das Banale sowie dessen kulturtheoretische Reflexion breiten Eingang in Abfall finden, ist bei einem Akteur der Popliteratur und insbesondere bei dessen literarischer Vorliebe für Medienprotokolle wenig überraschend. ${ }^{47}$ Vorwiegend gerahmt von häuslichen Tätigkeiten wie Aufräumen oder Duschen finden sich Lektürenotizen zu Tagespresse, Fernsehen und Büchern aller Zeiten. Dabei fasziniert den Diaristen insbesondere die Gleichzeitigkeit

44 Rainald Goetz, Abfall für alle. Roman eines Jahres [Internetveröffentlichung 1999], Frankfurt a.M. 2003, I, S. 13; S. 37.

45 Ebd., 7.7.7, S. 863.

46 Ebd., III/1.5, S. 313.

47 Vgl. Lutz Hagestedt, ... und gehalten alles nur von der Strenge der Zeit. Rainald Goetz als Tagebuch-Autor, in: Helmut Gold/Christiane Holm/Eva Bös/Tine Nowak (Hg.), @bsolut privat?, S. 108-111. 
solcher Lektüren: »Und dann speist sich das zurück, über den eigenen Kopf, in die Realität des Diskurses, via Abfall zum Beispiel, so wie hier. ${ }^{48}$ Der Schreibende entwirft sich somit nicht als diskursprägender Autor, sondern als Nachverwerter.

Wenngleich Goetz sich vehement gegen die metaphorische Rede verwehrt, so bietet Abfall einer interpretationsfreudigen Lektüre, die die motivische Präsenz von Müll kaum übersehen kann, einiges an: So ist an einem Montag, wie eben zitiert, der diskursive Abfall, an einem Sonntag der häusliche Abfall Gegenstand des Diariums:

Armut: man fängt an mit zwei Fingern im halbvollen Staubsauger-Papiersack nach den Staubballungen zu tasten, ob man das Ding schon wegschmeißen muß, oder ob es eigentlich noch geht, gehen müßte, obwohl die Saugkraft schon so schwach ist, beim laufenden Staubsauger usw usw, und ehe man sich versieht, puhlt man da so konzentriert wollige Staubballen aus dem engen Staubansaugeloch, wie so ein Gestörter, total Kranker - wirklich Armut? Oder ist das nicht eher schon Geiz, Angst, Angstgeiz, totale Perversion? Kaputte Szene. Krank. Große Haushaltsoper, riesen $\mathrm{Spaß} .^{49}$

Der Diarist als Wissens-Staubsauger, der bei nachlassender Saugkraft am siebten Tage seinen Papiersack leert, und ein höchst verdichtetes DiskursKonzentrat vorfindet, das sich unmerklich in ihm formiert hat. Insofern enthält das Tagebuch das, was an Wissen hängen blieb.

Der Grund für die Wahl des Onlineformates liegt nicht, wie der Diarist ausführt, in dessen interaktiven Möglichkeiten, die bezeichnenderweise völlig ungenutzt bleiben: Der Text ist weder verlinkt noch können die Leser sich einschalten. Die Entscheidung für die Form gründet allein auf dem real time effect, so dass die jeweilige Texteinheit >Tag täglich und nicht erst als Tagebuch im Sinne eines Textganzen erscheint. Die Tages-Einträge sind zudem durch den "ZIFFERNWAHNSINN" der Minuten- und Sekundenangaben strukturiert. ${ }^{50}$ Diese Untereinheiten, die sogenannten "Minutendinger", werden als zentrales Kriterium für die Unterscheidung von den in großer Zahl rezipierten gedruckten Tagebüchern herangezogen. ${ }^{51} \mathrm{Im}$ Verlauf des Diariums verstärken sich die Überlegungen zur Reflexion dieser Zeitstruktur auch durch den Einschub der Praxis-Skripte.

48 Rainald Goetz, Abfall für alle, I/3.1, S. 55f.

49 Ebd., I/3.7., S. 67.

50 Ebd., I, S. 14.

51 Ebd., I, S. 16. 
Wo also ist der Ort des Textes? Abstrakt gesprochen [...] steht der Text im Moment der Entstehung am denkbar AUSGESETZTESTEN Ort: im Jetzt.

Harrend auf das Kommende des nächsten Moments, des jeweils nächsten Augenblicks, des von daher kommenden, darin sich gebenden nächsten Worts.

Um den Text aufnehmen zu können, muss man sich selbst an diesen Ort begeben. Und wenn es etwas gibt, was einen am Schreiben, so absolut das Allerschönste es auch ist, trotzdem zuinnerst und brutalst FERTIG macht, dann diese Position. Sie bestimmt das Leben. ${ }^{52}$

Das Tagebuch ermöglicht dieses poetologische Konzept in radikaler Weise, weil es die bedingungslose Einlassung auf die Gegenwart provoziert. Und es entwirft eine Praxis, die weder dem retrospektiven Archivieren noch dem prospektiven Bilanzieren entspricht, sondern einer Form des Navigierens, die den Ort als Zeitpunkt definiert. Entsprechend diesem diaristischen Scripteur soll auch der Leser von Abfall kein Wissens-Staubsauger zweiter Ordnung sein, sondern an der Praxis partizipieren: ${ }^{.3}$ "Meine Lieblingsvorstellung vom Effekt dieser Täglichkeit einer so abstrakten Nähe ist das Verschwinden des Textes im Leben des Lesers. Daß sich vielleicht nur ein leicht gesteigertes Gefühl für die eigene Zeit ergeben würde. «" ${ }^{54}$

Die konsequenteste Umsetzung dieser Navigation im Gegenwärtigen ist, und dies ist mehrfach Thema, das Notieren von Zeitzahlen, bezeichnenderweise erfolgt es am intensivsten zum Jahreswechsel.

Donnerstag, 31.12.98, Berlin.

1111.07.

1111.11 .

1111.33 .

1111.46.

1111.51 .

1111.58 .

1112.03 .

1112.13 .

1112.14 .

1112.41. Chronist des Augenblicks. Ich wollte mal einen Tag lang hier nur Zeitziffern notieren, einfach so, jedesmal wenn ich auf die Uhr schaue, weil das so schön

52 Ebd., PRAXIS IV, S. 328

${ }^{53} \mathrm{Zu}$ den Verfahren der digitalen Textnavigation: Natalie Binczek, »Wo also ist der Ort des Textes? « - Rainald Goetz' Abfall fuir alle, in: Peter Gendolla/Norbert Schmitz/Irmela Schneider/Peter Spangenberg (Hg.), Interaktive Medienkunst, Frankfurt a.M. 2001, S. 291-318.

54 Rainald Goetz, Abfall für alle, 7.7.7, S. 864. 
ausschaut, nur diese Ziffern, die so viel bedeuten, aber letztlich sind mir solche experimentellen Kaspereien dann eben doch immer wieder nach kürzester Zeit zu blöd. ${ }^{55}$

\section{Résumé}

Es ist die vom Tagestakt disponierte Gebrauchsform, über die sich das diaristische Verhältnis zum Unnützen Wissen herstellt. Die drei Tagebücher von Lavater, Schnitzler und Goetz sind nicht nur einschlägig für das unsichere Wissen vom Menschen, sondern sie können darüber hinaus zeigen, dass das Unnütze Wissen ein Effekt der Praxis ist, da es im Schreibprozess immer wieder umdefiniert und somit beobachtbar wird.

Der Vergleich von Lavaters beiden bilanzierenden Tagebüchern kann zeigen, wie die aus dem theologischen Wissen übertragene normative Unterscheidung von Nutz und Unnutz zunehmend von einem sich konstituierenden diaristischen Praxiswissen korrigiert wird, infolge dessen Nutz und Unnutz an Relation und Prozess ausgerichtet werden. Schnitzlers archivierendes Tagebuch führt am Traum als dem zentralen Ort psychoanalytischer Theoriebildung vor, dass gerade die Vergegenwärtigung solcherart Tagesreste, die weniger in der Tiefe des Subjekts als in der Oberfläche des Alltags gründen, eine diaristische Traumdeutung plausibilisieren kann, die dem Banalen eine Schlüsselposition einräumt. Und Goetz geht es in seinem momentanistischen Konzept des aktuell nicht Brauchbaren, des Minuten-Abfalls, darum, sich in emphatischer Weise der Gegenwart auszuliefern, um darauf eine Poetik zu begründen.

55 Ebd., VII/6.4, S. 833 
